\title{
EFEITOS DE CONJUNTOS DE DICAS EM TAREFAS DE DISCRIMINAÇÃO CONDICIONAL ${ }^{1}$
}

\author{
Thalita Filier Cazetto* \\ Ana Carolina Sella
}

\begin{abstract}
RESUMO. Discriminações condicionais possibilitam que o ensino de alguns comportamentos derive em outros sem que tenham de ser diretamente ensinados. Apesar da suposta economia de ensino, dificuldades têm sido relatadas no estabelecimento do controle adequado de estímulos nestas discriminações. A utilização de dicas como demonstrações e/ou instruções pode auxiliar no estabelecimento de tal controle de estímulos. O objetivo do presente estudo foi avaliar se haveria diferenças de desempenho diante da apresentação de dois conjuntos de dicas. Participaram do estudo 20 crianças de idades entre 9 e 12 anos, distribuídas em dois grupos. Ao primeiro grupo foi apresentado um procedimento de escolha de acordo com o modelo, adicionado a dicas (demonstração e instrução) acerca do estímulocomparação correto. Ao segundo grupo foi apresentado o mesmo procedimento, porém as dicas eram acerca dos pares de relações condicionais. Os resultados mostraram que as dicas acerca dos pares de relações condicionais geraram melhores desempenhos.
\end{abstract}

Palavras-chave: escolha de acordo com o modelo, crianças, dicas.

\section{EFFECTS OF PROMPTS SETS IN CONDITIONAL DISCRIMINATIONS TASKS}

\begin{abstract}
Conditional discriminations allow that by teaching some behaviors, others will emerge without having to be directly taught. Despite the supposed teaching economy, difficulties have been reported in the establishment of the adequate stimulus control in such discriminations. The use of prompts, such as demonstrations and/or instructions, can help establishing the adequate stimulus control. The purpose of the present study was to evaluate whether there would be differences in performance when two sets of prompts were presented. The study included 20 children aged between 9 and 12 years divided into two groups. To the first group, a matching to sample task was presented added to prompts (demonstration and instruction) about the correct comparison stimulus. To the second group the same procedure was presented, but the prompts were about the conditional relation pairs. The results showed that the prompts about the conditional relation pairs yielded better performances.
\end{abstract}

Key words: Matching to sample,children, prompts.

\section{EFECTOS DE CONJUNTOS DE PROMPTS EN TAREAS DE DISCRIMINACIÓN CONDICIONAL}

\begin{abstract}
RESUMEN. Discriminaciones condicionales permiten que la enseñanza de algunas conductas se derive en otras sin tener que ser directamente enseñadas. A pesar de la supuesta economía en la enseñanza, dificultades han sido reportadas en el establecimiento del control de estímulos adecuado en este tipo de discriminación. El uso de prompts, como por ejemplo, demostraciones e/o instrucciones, puede ayudar a establecer el control de estímulos adecuado. El objetivo de este estudio fue evaluar si había diferencias en el rendimiento cuando presentados dos conjuntos de prompts. Participaron del estudio 20 niños, edad entre 9 y 12 años, divididos en dos grupos. Al primer grupo se presentó un procedimiento de igualación de la muestra añadido de prompts (demostración e instrucciones) acerca del estímulo de comparación correcto. Al segundo grupo se presentó el mismo procedimiento, pero los prompts eran acerca de los pares de las relaciones condicionales. Los resultados mostraron que los prompts acerca de los pares de las relaciones condicionales genero un mejor rendimiento.
\end{abstract}

Palabras-clave: Igualación de la muestra, niños, prompts.

\footnotetext{
Apoio: CNPq.

Psicóloga formada pela Universidade Federal de São Carlos, Brasil (2010).

\# Doutorado em Educação Especial (Educação do Indivíduo Especial) pela Universidade Federal de São Carlos, Brasil (2009).

Colaboradora da Universidade de Ciências da Saúde de Alagoas, Brasil.
} 
As chamadas discriminações condicionais têm sido bastante estudadas, por fornecerem ferramentas para o entendimento de comportamentos humanos complexos, por sua aplicabilidade no ensino de habilidades acadêmicas e por possibilitarem que, do ensino direto de algumas habilidades, outras emerjam sem ser necessário reforçá-las ou ensiná-las diretamente (K. Saunders, R. R. Saunders, Williams \& Spradlin, 1993; K. Saunders \& Spradlin, 1989; Sidman, 1986, 1990, 1992).

Apesar dos resultados positivos que vêm sendo obtidos com o uso de procedimentos de ensino de discriminações condicionais, mais especificamente com o procedimento de escolha de acordo com o modelo (Sidman, 1971; Sidman \& Cresson, 1973; entre outros), diversos estudos têm documentado dificuldades no ensino de tais discriminações (Eikeseth \& Smith, 1992; K. Saunders \& Spradlin, 1989, 1993; Pilgrim, Jackson \& Galizio, 2000). Os principais problemas encontram-se no estabelecimento do controle de estímulos adequado para essas discriminações. Green, Sigurdardottir e R. Saunders (1991), Michael e Bernstein (1991) e Piccolo (2004) sugerem que a utilização de dicas ${ }^{2}$ como, por exemplo, demonstrações e/ou instruções, pode auxiliar no ensino de discriminações condicionais.

O uso de demonstrações como dicas no ensino de discriminações teve início na década de 80 , porém os estudos até agora realizados ainda não apresentam uma sistematização de procedimentos e os resultados obtidos ainda são controversos.

O primeiro estudo que utilizou demonstrações como dica para o ensino de discriminações condicionais foi realizado por MacDonald, Dixon e LeBlanc (1986). Os autores ensinaram quatro pares de discriminações condicionais entre os conjuntos de estímulos denominados A, B e C (os quais eram compostos por símbolos agrícolas) a quatro participantes com deficiência mental. Cada participante aprendeu um par de discriminações por ensino direto e um segundo par pela observação do outro participante (ensino por meio de demonstração). Durante o ensino direto era apresentada a instrução "Este (e o estímulo-comparação correto era apontado) vai com o do topo (e apontava-se o estímulo-

2 A palavra "dica" é aqui utilizada como a tradução da palavra inglesa prompt. Prompt é definido como um estímulo antecedente, como por exemplo, uma instrução, um modelo, ou algum tipo de ajuda física que a pessoa responsável pelo ensino apresenta/utiliza para obter uma resposta do aprendiz que possa ser reforçada dentro do contexto de ensino (Demchak, 1990). amostra)". Dois participantes aprenderam relações $\mathrm{BA}$, enquanto dois aprenderam CA por ensino direto. A seguir, no ensino por demonstração, colocava-se um participante que havia aprendido a relação BA com um que aprendera CA. Enquanto um dos participantes executava tentativas relativas àquelas que aprendera por ensino direto, o outro apenas observava. Aquele que observa tinha a oportunidade de responder apenas após a demonstração de todas as tentativas por um participante. Respostas corretas em tarefas que foram observadas resultavam em consequências programadas. Todos os participantes aprenderam as relações ensinadas por ensino direto e apenas um participante aprendeu imediatamente as relações ensinadas por demonstrações. Foram então apresentadas novas dicas aos três participantes que não haviam aprendido as relações condicionais por demonstrações, a saber, instruções mínimas a respeito da tarefa a ser executada. O experimentador dizia: "Aponte para aquele que (nome do companheiro) iria apontar se ele visse esta figura". Dois dos três participantes responderam de acordo com o esperado após a inserção das novas dicas, mas o terceiro participante não alcançou o critério de desempenho.

Michael e Bernstein (1991) compararam o efeito de demonstrações, instruções verbais e modelagem em tarefas de discriminações condicionais. Participaram do estudo três grupos de crianças (cinco crianças no grupo das demonstrações, quatro no grupo das instruções verbais e três no grupo da modelagem). No tocante ao grupo ao qual foram apresentadas instruções verbais, estas diziam respeito à tarefa que seria realizada e aos pares de estímulos que deveriam "ir juntos" para formarem a resposta correta. Ao grupo exposto à condição de demonstração era apresentada uma instrução verbal mínima para o participante observar a demonstração. Na condição de modelagem, uma instrução simples era apresentada acerca da necessidade de tocar a tela do computador. Respostas corretas resultavam em consequências programadas para todos os grupos. Tanto o grupo exposto às instruções detalhadas quanto aquele exposto à demonstração aprenderam as discriminações condicionais mais rapidamente do que aqueles que foram expostos à modelagem.

Piccolo (2004) investigou o efeito de demonstrações sobre a aprendizagem de discriminações condicionais a partir da realização de diversos estudos. No estudo que a autora denominou Experimento 4, primeiramente foram ensinadas relações BA por ensino direto a oito 
crianças pré-escolares. Consequências foram programadas para respostas corretas e incorretas ao longo do ensino destas relações: Respostas corretas produziam a apresentação de fichas e "reforço social" pelo experimentador, som emitido pelo computador e contraste figura-fundo na tela. Respostas incorretas resultavam em a tela do computador se tornar branca e em um intervalo de dois segundos entre as tentativas. Todos os participantes aprenderam as discriminações condicionais apresentadas. Em seguida foram ensinadas relações CA por demonstração, sem consequências programadas. Nesta fase do estudo, o participante observava um demonstrador executando um bloco de tentativas. Assim que todas as demonstrações terminavam, era dada ao partcipante a oportunidade de responder a tentativas semelhantes àquelas demonstradas. Apenas um participante não aprendeu estas novas relações. Os conjuntos $\mathrm{A}, \mathrm{B}$ e $\mathrm{C}$ eram compostos por figuras familiares monocromáticas. Em outro de seus estudos de 2004, denominado Experimento 5, Piccolo procurou investigar a efetividade de demonstrações sem que nenhuma discriminação condicional fosse ensinada de forma direta. Para avaliar se as demonstrações por si sós seriam efetivas, após cada resposta do participante, correta ou incorreta, eram apresentados apenas o intervalo entre as tentativas e a próxima tentativa. Participaram deste estudo sete pessoas, com idades entre 11 e 15 anos, que frequentavam uma sala de educação especial. Foram utilizadas figuras familiares monocromáticas como estímulos experimentais. Três dos sete participantes alcançaram o critério de desempenho nas relações ensinadas (BA e CA) sem a presença de reforço diferencial a cada tentativa. Todos os outros participantes precisaram de instruções e dicas adicionais para aprender as relações BA e CA.

Buscando estender a investigação de Piccolo (2004), Sella (2005) investigou se a apresentação de demonstrações de discriminações condicionais em blocos de 12 tentativas ou em tentativa a tentativa influenciaria a aprendizagem de tais discriminações em uma população de crianças com deficiência intelectual. Buscando isolar variáveis relacionadas à familiaridade dos participantes com os estímulos, Sella (2005) utilizou figuras monocromáticas abstratas presentes no programa computacional Spell ® (Dube \& McIlvane, 1989). Nos blocos de tentativas, o experimentador executava 12 tentativas seguidas e na sequência o participante executava seu bloco de 12 tentativas.
Quando as tentativas eram apresentadas uma a uma, a cada tentativa demonstrada pelo experimentador $\mathrm{o}$ participante executava uma tentativa. Quantitativamente, os resultados apontaram para uma igualdade de efetividade das duas formas de apresentação de demonstrações: ambas as formas de apresentação resultaram em uma média de 20 sessões para alcance de critério de desempenho nas discriminações condicionais ensinadas. Por outro lado, no aspecto qualitativo pôde-se observar que foram diferentes os controles de estímulos entre uma e a outra forma de apresentação de demonstrações. Quando as demonstrações eram apresentadas uma a uma, o comportamento dos participantes parecia ficar sob controle apenas das escolhas do estímulo-comparação feitas pelo demonstrador: o desempenho dos participantes caía durante testes em que as demonstrações não estavam presentes, ou seja, o comportamento dos participantes não ficava sob controle da relação condicional. Quando as demonstrações eram apresentadas em bloco, o desempenho dos participantes não caía durante os testes sem demonstração, porém era necessária a apresentação de mais sessões de ensino para as respostas ficarem sob o controle das discriminações condicionais esperadas.

Buscando-se estender os experimentos anteriormente descritos, o objetivo do presente estudo foi verificar se há diferenças de desempenho quando instruções acerca dos pares de relações condicionais nas tarefas de escolha de acordo com o modelo são adicionadas a demonstrações ou quando instruções apenas referentes ao estímulo-comparação correto são adicionadas as demonstrações.

\section{MÉTODO}

\section{Participantes}

Participaram do estudo 20 crianças da quarta série do Ensino Fundamental, que tinham entre 9 e 12 anos de idade e frequentavam uma escola estadual da cidade de São Carlos, São Paulo. Estas crianças foram distribuídas igualmente entre dois grupos. Participaram da pesquisa apenas aquelas crianças cujos pais, através de termos de consentimento livre e esclarecido, autorizaram sua participação.

\section{Ambiente e materiais experimentais}

A pesquisa foi realizada em uma sala de aula cedida pela escola dos participantes. Diariamente, 
duas carteiras e duas mesas foram posicionadas lado a lado, na parte anterior da sala, próximas ao quadronegro, de forma que o experimentador pudesse sentarse ao lado do participante enquanto este realizava as tarefas. O computador foi alocado sobre a mesa posicionada à frente do participante. As sessões foram conduzidas três vezes por semana e duravam entre três e dez minutos. Diariamente foram conduzidas, em média, três sessões individuais.

Foi utilizado um computador do tipo portátil acompanhado de um mouse óptico. Neste computador foi instalado o programa computacional Mestre ${ }^{\circledR}$ (Goyos \& Almeida, 1994), que permitiu a programação e registro dos dados no procedimento de escolha de acordo com o modelo.

Foram também utilizados jogos de tabuleiro diversos, uma lata amarela, uma lata verde, bolas vermelhas, bolas amarelas, cubos vermelhos, cubos amarelos, cilindros vermelhos, cilindros amarelos e uma tigela.

\section{Estímulos experimentais}

Seis figuras abstratas foram apresentadas em linha preta sobre um fundo branco de cerca de $7 \mathrm{~cm} \mathrm{X} 7 \mathrm{~cm}$. As figuras eram similares àquelas do software de pesquisa Spell ${ }^{\circledR}$ (Dube \& McIlvane, 1989) e foram distribuídas aleatoriamente entre dois grupos de estímulos, $\mathrm{A}_{1,2,3}$ e $\mathrm{B}_{1,2,3}$. As figuras encontram-se representadas na figura 1 .

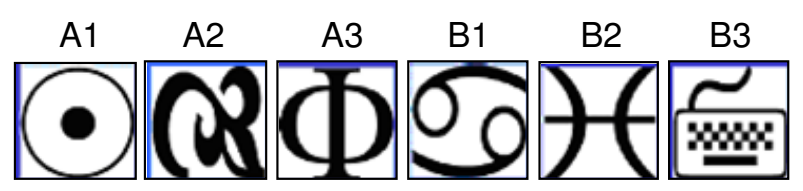

Figura 1. Estímulos Experimentais

\section{Variável dependente}

A variável dependente de interesse foi a porcentagem de acertos em tarefas de discriminação condicional.

\section{Fidedignidade}

Os dados acerca do desempenho dos participantes foram registrados pelo computador. O software utilizado já havia sido calibrado em relação à fidedignidade de seu registro. Em relação à fidedignidade do procedimento, ou seja, à apresentação das instruções pelo experimentador, $20 \%$ das sessões foram filmadas e avaliadas por um segundo observador. Houve $100 \%$ de acordo em relação à presença das instruções, conforme descritas abaixo.

\section{Procedimento geral}

Primeiramente foi realizada uma identificação de jogos de preferência para estabelecer possíveis jogos que poderiam servir como itens reforçadores ao longo do experimento. Em seguida estabeleceram-se moedas como reforçadores condicionados, ao mesmo tempo em que se realizou um teste de seguimento de instrução para verificar se os participantes tinham estas respostas em seu repertório. Estes dois procedimentos encontram-se descritos na íntegra em Sella (2005). Logo após este passo, foram ensinadas três discriminações condicionais (A1B1, A2B2 e A3B3) através de tarefas de acordo com o modelo, as quais incluíam as demonstrações e instruções apresentadas pelo computador. Para cada grupo de crianças, as demonstrações possuíam as peculiaridades descritas abaixo.

\section{Tarefas de escolha de acordo com o modelo}

No início de cada sessão a experimentadora dizia: "O computador vai te ensinar a jogar. Cada vez que você acertar, vai ganhar moedas e, no final de cada jogo, vai poder trocar suas moedas por uma partida do jogo que você queira. A cada nove moedas que você ganhar, poderá jogar uma partida do jogo que quiser. As moedas que sobrarem não poderão ser usadas em outro dia". As tarefas de escolha de acordo com o modelo eram iniciadas com a apresentação de um estímulo-amostra na parte superior da tela do computador. Na primeira tentativa da primeira sessão o computador apresentava uma instrução para que o participante clicasse com o mouse sobre este estímulo. Após o toque sobre este estímulo, três pontos de interrogação apareciam no canto superior direito da tela do computador e três estímulos-comparação apareciam na parte inferior da tela do computador. O computador apresentava a instrução de que o participante clicasse em um dos pontos de interrogação. Ao clicar em um destes pontos, a cor do fundo da tela mudava e apresentava-se uma demonstração de uma tentativa da tarefa. Para cada grupo foi apresentado um tipo de demonstração. Demonstrações eram repetidas mais duas vezes nesta primeira sessão. Conforme o participante clicava sobre os botões de ajuda, estes desapareciam, restando apenas aqueles que ainda poderiam ser utilizados. Nas próximas sessões, a única instrução apresentada versava sobre a possibilidade de a criança pedir demonstrações em três tentativas, quando e se o desejasse. As sessões de tarefas de escolha de acordo com o modelo foram compostas em geral por 18 tentativas. Nas 
sessões em que as demonstrações não eram obrigatórias, este número poderia variar entre $15 \mathrm{e}$ 18 tentativas, dependendo do número de demonstrações pedidas pelo participante. $\mathrm{O}$ critério de desempenho de ambos os grupos foi a emissão de 9 respostas corretas consecutivas, sem pedido de demonstração, em uma única sessão. Se não alcançado o critério de desempenho dentro das 10 sessões previstas, o participante era desligado do estudo. A previsão de 10 sessões foi feita a partir dos dados de Sella (2005).

\section{Instrução com indicação apenas do estímulo- comparação correto (Grupo 1)}

Para este grupo, além das instruções apresentadas pela experimentadora, a seguinte instrução era apresentada pelo computador na primeira sessão: "Esta é uma tentativa para mostrar para você como é que se joga (aparecia, então, um estímulo-amostra na parte superior da tela do computador). Toque esta figura (apareciam três estímulos-comparação na parte inferior da tela e três botões de ajuda, representados por pontos de interrogação, à direita do estímulo-modelo, no topo da tela). Agora você pode pedir ajuda tocando um ponto de interrogação. Toque um ponto de interrogação (a tela mudava a cor de seu fundo, os estímulos-comparação tinham suas posições modificadas e uma seta aparecia sobre o estímulocomparação correto). A figura com a seta em cima é a correta. Toque esta figura (após o toque sobre a figura, tanto a cor do fundo da tela como as posições dos estímulos-comparação voltavam a seu aspecto anterior ao toque sobre o ponto de interrogação). Agora tente uma, sozinho". A intercalação entre demonstração e execução pelo participante se repetia mais duas vezes nesta primeira sessão. A partir da segunda sessão, a instrução apresentada pelo computador no início da sessão era: "Você está aqui para jogar no computador. Não esqueça que você pode pedir ajuda três vezes para o ponto de interrogação sobre como é que se joga (apareciam, então, o estímulomodelo e os três botões de ajuda). Toque esta figura e pode começar". Cada vez que o participante tocava um botão de ajuda, uma demonstração da resposta correta era apresentada, conforme descrito anteriormente. Até três demonstrações poderiam ser apresentadas em uma sessão.

\section{Instrução com indicação de relação condicional} correta (Grupo 2)

Para este grupo foi utilizado o mesmo procedimento do Grupo 1, mudando-se apenas parte da instrução apresentada durante as demonstrações. A primeira sessão tinha início com a apresentação da seguinte instrução pelo computador: "Esta é uma tentativa para mostrar para você como é que se joga (aparecia, então, um estímulo-amostra na parte superior da tela do computador). Toque esta figura (após o toque, apareciam três estímuloscomparação e três botões de ajuda à direita, no topo da tela). Agora, você pode pedir ajuda tocando um ponto de interrogação. Toque um ponto de interrogação (a cor do fundo da tela era modificada, assim como a posição dos estímulos-comparação; uma seta aparecia, ao mesmo tempo, sobre o estímulo-amostra e sobre o estímulocomparação designado como correto). Estas figuras sempre vão juntas. Juntas formam a resposta correta. Toque a figura de baixo (a cor da tela e as posições dos estímulos-comparação voltavam a seu aspecto anterior). Agora tente uma, sozinho". Como para o Grupo 1, mais duas tentativas com demonstrações eram apresentadas nesta primeira sessão. A partir da segunda sessão as instruções eram semelhantes àquelas apresentadas ao Grupo 1, excetuando-se as instruções dadas durante as demonstrações, as quais eram similares às descritas anteriormente.

\section{RESULTADOS}

Os resultados abaixo apresentados foram elencados na seguinte ordem: primeiramente, apresentaram-se as diferenças encontradas nos resultados entre os grupos e, posteriormente, resultados específicos acerca do desempenho dos participantes.

A figura 2 mostra as porcentagens de acertos, por sessões, dos participantes do Grupo 1, os quais receberam instruções apenas acerca do estímulocomparação correto durante as demonstrações, no ensino das relações A1B1 (barras escuras), A2B2 (barras cinza) e A3B3 (barras brancas). Dos 10 participantes do grupo 1 , quatro alcançaram critério de desempenho. Para 1A foi necessária uma sessão, para 1B foram necessárias três sessões e os grupos 1C e 1D alcançaram critério em seis sessões; e seis participantes não alcançaram o critério de desempenho: 1E, 1F, 1G, 1H, 1I e 1J. 
$1 \mathrm{~A}$

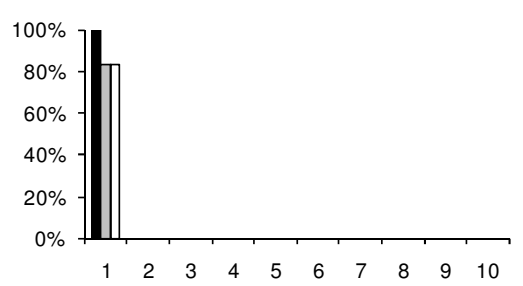

$1 C$

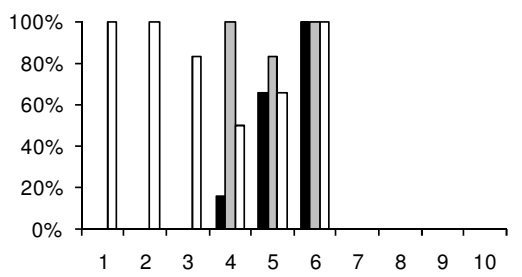

$1 E$

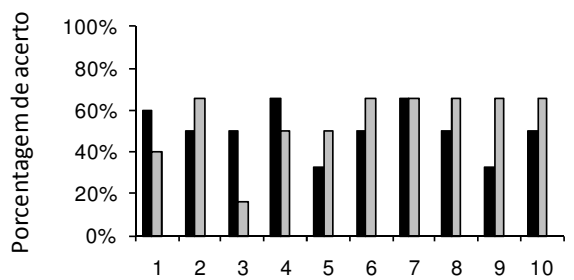

$1 G$

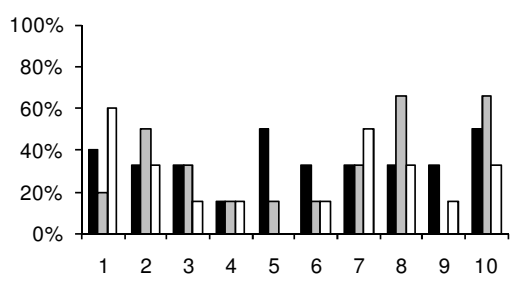

11

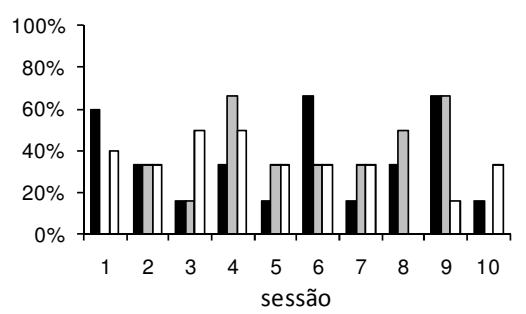

1B

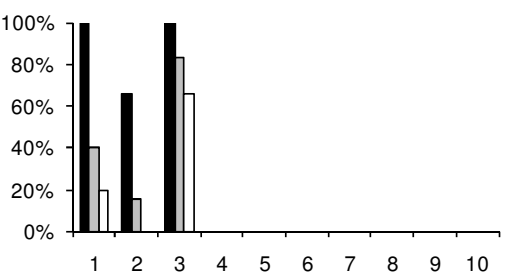

1D

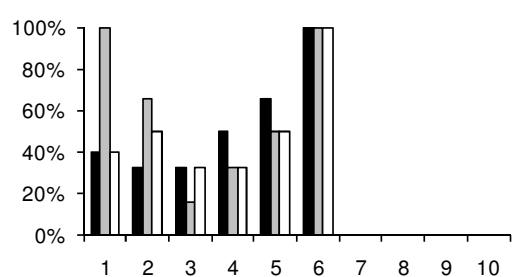

1F

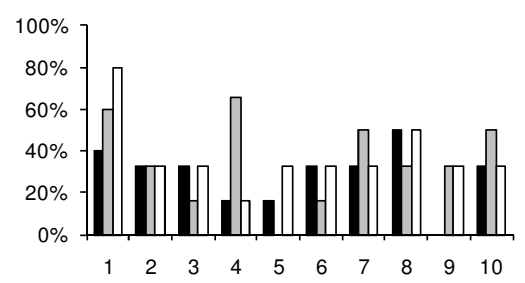

1H

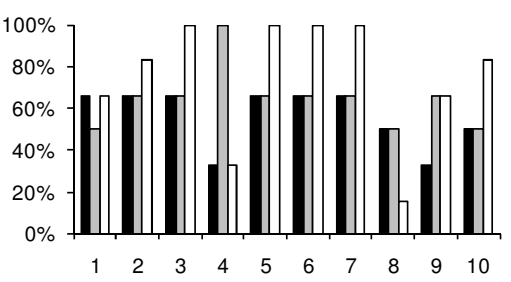

1J

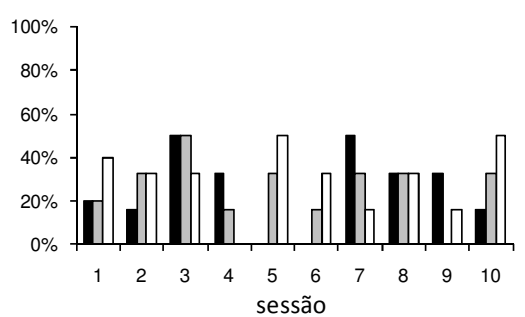

Figura 2. Porcentagem de Acertos, por Sessão, dos Participantes do Grupo 1. As Barras Escuras Indicam os Resultados Relativos ao Ensino da Relação A1B1; as Barras Cinza Indicam os Resultados Relativos ao Ensino de A2B2, e as Barras Brancas Indicam os Resultados Relativos ao Ensino de A3B3.

A Figura 3 mostra as porcentagens de acertos por sessões dos participantes do grupo 2, os quais receberam instruções acerca dos pares de relações condicionais nas demonstrações, no ensino das relações A1B1 (barras escuras), A2B2 (barras cinza) e A3B3 (barras brancas). No grupo 2, nove dos 10 participantes atingiram o critério de desempenho dentro das 10 sessões estabelecidas. Três participantes 
alcançaram o critério de desempenho logo na primeira sessão (participantes 2A, 2B e 2C), dois o alcançaram critério na terceira sessão de ensino (2D e $2 \mathrm{E}$ ) e três o alcançaram na quarta sessão de ensino: $2 \mathrm{~F}, 2 \mathrm{G}$ e $2 \mathrm{H}$.

$2 A$

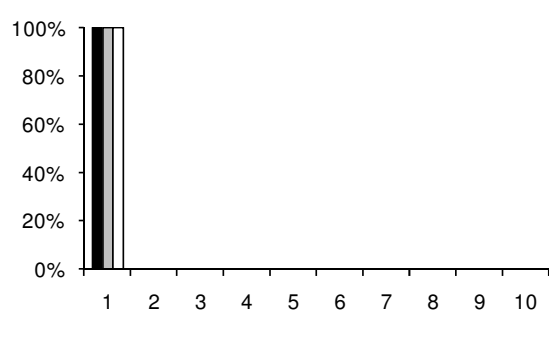

2C

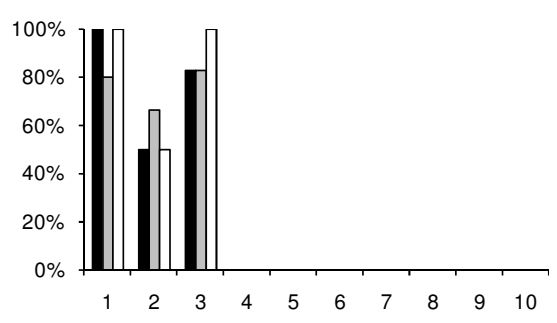

2E

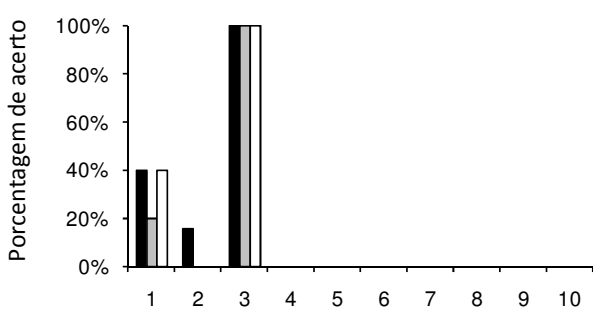

$2 \mathrm{G}$

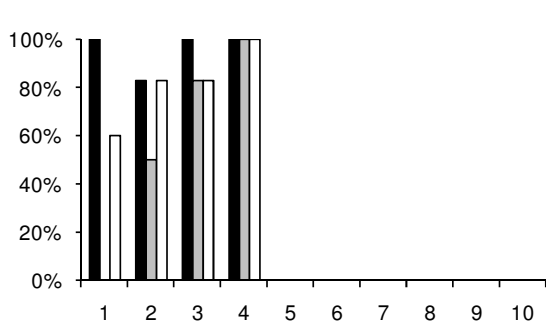

2I

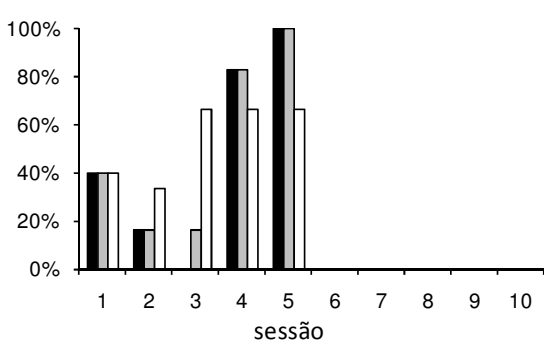

O participante 2I atingiu o critério de desempenho na quinta sessão de ensino, não tendo emitido respostas incorretas nesta sessão. Um participante, 2J, não atingiu o critério de desempenho.

2B

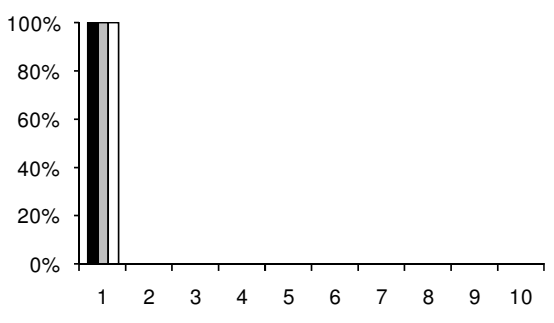

2D

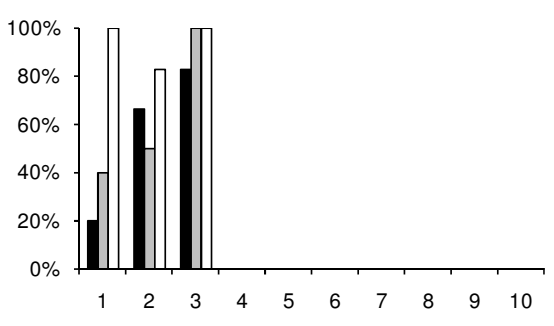

$2 F$

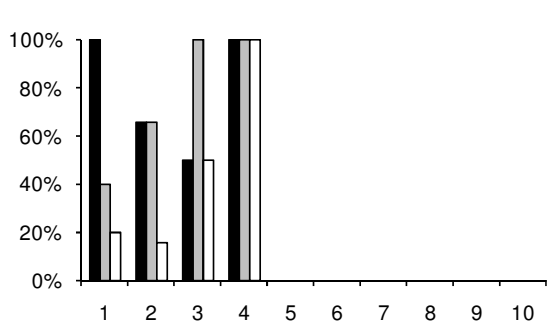

2H

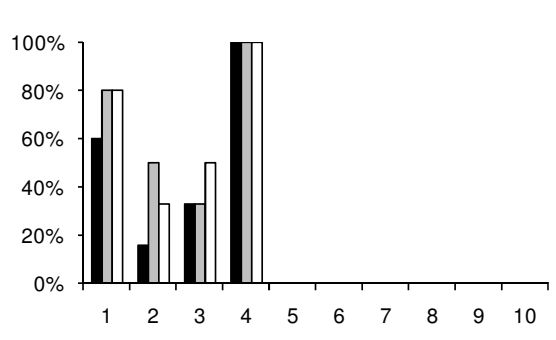

2J

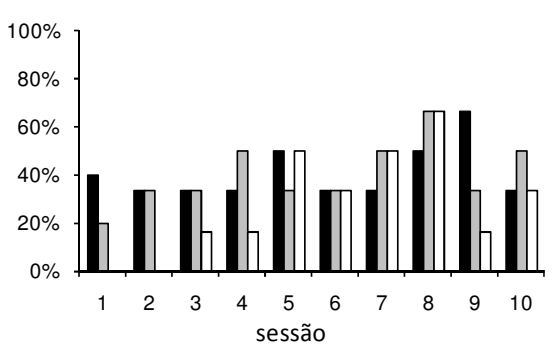

Figura 3. Porcentagem de Acerto por Sessão dos Participantes do Grupo 2. As Barras Escuras Indicam os Resultados Relativos ao Ensino da Relação A1B1; as Barras Cinza Indicam os Resultados Relativos ao Ensino de A2B2, e as Barras Brancas Indicam os Resultados Relativos ao Ensino de A3B3. 
Comparando-se os desempenhos representados na figura 2 e na figura 3, observa-se que as diferentes instruções resultaram em desempenhos diferentes entre os dois grupos. De forma geral, quase todos os participantes do grupo 2 alcançaram o critério de desempenho, enquanto apenas quatro participantes do Grupo 1 o fizeram. Apesar de mais participantes terem alcançado o critério de desempenho quando foram dadas instruções acerca dos pares de relações condicionais (grupo 2), é importante ressaltar que para um participante do grupo 2 esta instrução não foi efetiva. Esta questão será retomada a seguir, devido à importância dos dados individuais quando se tem em vista a utilização de delineamentos de sujeitos únicos (Sidman, 1960; entre outros).
Ao serem analisadas de forma mais específica as respostas incorretas emitidas pelos participantes foram observados diferentes tipos de controle por estímulos não programados nas contingências do experimento, a saber, controle por posição, preferência por estímulo e escolha de uma determinada sequência de estímulos. Os dados mostram que a escolha de uma determinada sequência de estímulos ocorreu quando 13 dos 20 participantes emitiram respostas consideradas incorretas. Participantes do grupo 1 emitiram mais respostas sob controle de estímulos espúrios do que participantes do grupo 2, conforme se pode visualizar na figura 4.

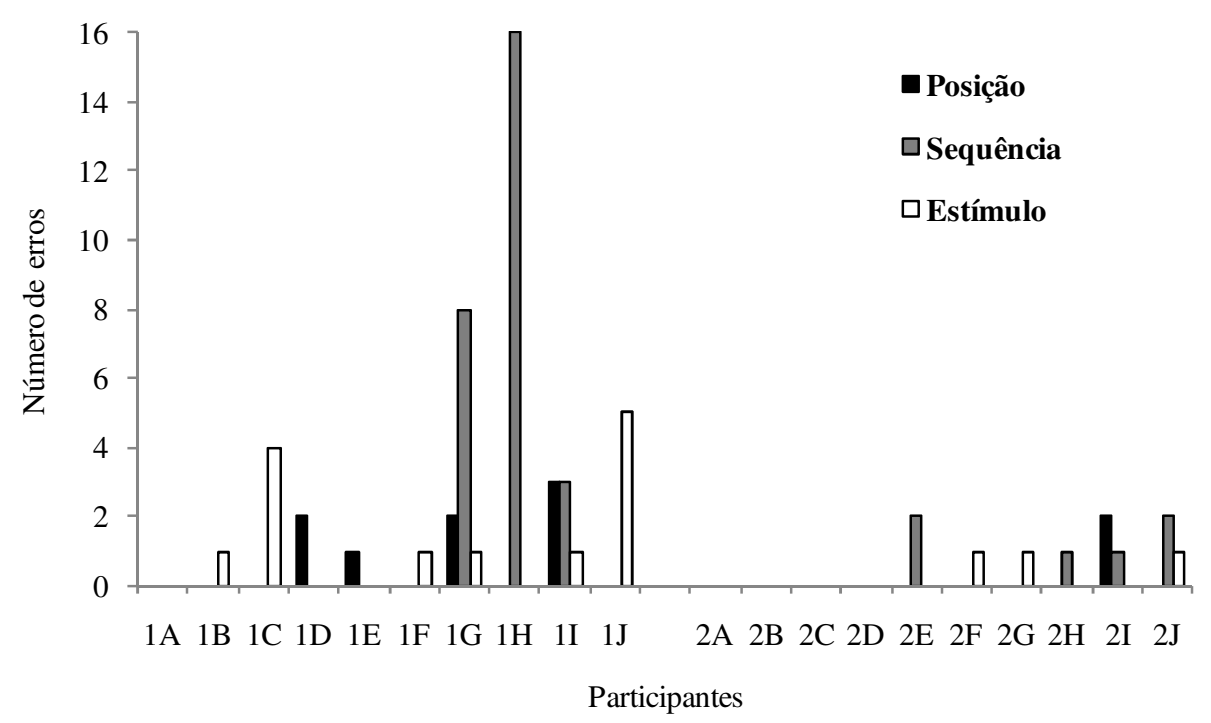

Figura 4. Número de Respostas Incorretas e Tipo de Controle Espúrio para Todos os Participantes.

\section{DISCUSSÃO}

Observando-se os resultados dos participantes e as diferenças entre os grupos, três aspectos parecem ser os mais relevantes a serem discutidos: o efeito das instruções apresentadas sobre o desempenho de cada um dos grupos; a dificuldade que foi encontrada em se estabelecer o controle de estímulos esperado para ambos os grupos; e a predominância do controle espúrio por determinadas sequências de estímuloscomparação.

Os dados mostram que as instruções acerca dos pares de discriminações condicionais apresentadas ao grupo 2 foram mais efetivas do que aquelas instruções apresentadas ao grupo 1, cujos participantes receberam instruções apenas acerca do estímulocomparação correto. Estes resultados vão ao encontro daqueles obtidos por Drake e Wilson (2008), Michael e Bernstein (1991) e Pilgrim et al. (2000), os quais mostram que instruções acerca dos pares de relações condicionais facilitam o desempenho em tarefas de escolha de acordo com o modelo.

Apesar de os resultados obtidos com as instruções acerca dos pares de relações condicionais (instruções para o grupo 2) terem sido melhores do que aqueles obtidos com as instruções apresentadas ao grupo 1, é importante ressaltar que, para alguns participantes, nenhum dos dois tipos de instrução resultou no alcance do critério de desempenho. A dificuldade em se estabelecer o controle de estímulos esperado, mesmo após a apresentação de instruções, tem sido extensamente relatada na literatura (Arntzen, 2004; McIlvane, Dube, Kledaras, Iennaco \& Stoddard, 1990; K. Saunders \& Spradlin, 1989, 1993; Touchette, 1971; entre outros). A principal dificuldade encontrada no ensino de tarefas de escolha de acordo com o modelo 
é que estas expõem o indivíduo a tarefas novas, que exigem a emissão de diversas respostas para que se chegue ao resultado esperado. Estas tarefas requerem discriminações simultâneas entre os estímuloscomparação apresentados em uma mesma tarefa, e sucessivas entre os estímulos-amostra nas diferentes apresentações da tarefa, para finalmente levar à discriminação condicional. Este tipo de tarefa, que envolve diversos estímulos e respostas, pode gerar relações estímulo-estímulo e estímulo-resposta que, em um determinado momento, vão inadvertidamente de encontro às contingências programadas, reforçando uma relação estímulo-resposta denominada controle espúrio (Cumming \& Berryman, 1961; Johnson \& Sidman, 1993; Sidman, 1987). Para superar esta dificuldade, a literatura de controle de estímulos propõe algumas soluções, como, por exemplo, a apresentação de dicas em forma de modelos e/ou instruções, conforme ocorreu no presente estudo; contudo, as dicas utilizadas não foram suficientes para conseguir os resultados esperados com todos os participantes. Para buscar procedimentos ainda mais efetivos, em que todos os participantes alcancem o critério de desempenho, outras formas de dicas, inclusive novas instruções, deveriam ser programadas e testadas para facilitar ainda mais a tarefa apresentada. Talvez seja necessário programar, inclusive, a retirada gradual de tais dicas, de forma que o controle do comportamento dos participantes passe gradualmente da dica para apenas os estímulos envolvidos na tarefa de escolha de acordo com o modelo em si, diminuindo a probabilidade de ocorrerem erros (Sidman \& Stoddard, 1967; Terrace, 1963, entre outros).

Os resultados da análise das respostas incorretas emitidas pelos participantes mostraram que respostas consideradas incorretas foram controladas principalmente por sequências de estímulos, ou seja, os participantes escolheram os estímulos-comparação em uma determinada sequência, independentemente dos estímulos-amostra apresentados. Este tipo de controle pode ter sido ocasionado pelo próprio procedimento de ensino: a presença de demonstrações nas três primeiras tentativas da primeira sessão pode, inadvertidamente, ter ensinado os participantes a responderem aos estímulos-comparação conforme a sequência demonstrada, sem ensinar-lhes que cada resposta estava condicionada à presença de um dado estímulo-amostra. Se as respostas de escolha dos participantes nestas três primeiras tentativas foram de encontro às contingências programadas e, de alguma forma, o controle de estímulos estabelecido não foi aquele programado pelo experimentador, os participantes podem ter aprendido a selecionar os estímulos de acordo com aquela sequência demonstrada, ao invés de terem aprendido a selecionar os estímulos-comparação de acordo com a presença de um dado estímulo-amostra. Este resultado é similar aos de Piccolo (2004) e Sella (2005), em que os comportamentos de diversos participantes ficaram sob controle apenas dos estímulos-comparação. Isto ainda pode ser ressaltado pelo fato de participantes do grupo 1 terem respondido sob controle de sequências de estímulos mais frequentemente do que participantes do grupo 2. Isto poderia ser esperado devido às instruções apresentadas ao grupo 1, nas quais apenas o estímulo-comparação correto era apontado na demonstração, em vez de ser apontada a relação condicional entre os estímulos. Conforme discute Sella (2005), a presença de demonstrações e/ou instruções que indiquem apenas o estímulo-comparação correto pode implicar que a resposta do observador fique sob controle apenas deste estímulo. Instruções e/ou demonstrações como as apresentadas ao grupo 2, nas quais se procura deixar as respostas dos participantes sob controle condicional explícito do estímulo-amostra e do estímulo-comparação, parecem reduzir a ocorrência de respostas sob controles espúrios.

\section{CONSIDERAÇÕES FINAIS}

A partir dos dados obtidos, infere-se que neste estudo a forma de apresentação das instruções ao longo das demonstrações foi uma variável que levou a diferentes desempenhos dos participantes. O desempenho do grupo 2 foi melhor que o do grupo 1, tanto em relação ao número de sessões para atingir o critério quanto em relação ao número de respostas incorretas emitidas pelos participantes. Apesar de tais resultados, devem ser programados novos estudos que considerem novas instruções e a presença de outras dicas além daquelas aqui utilizadas, considerando-se o fato de ter havido em ambos os grupos crianças que não alcançaram o critério de desempenho.

\section{REFERÊNCIAS}

Arntzen, E. (2004). Probability of equivalence formation: familiar stimuli and training sequence. The Psychological Record, 54, 275-291.

Cumming, W. W., \& Berryman, R. (1961). Some data on matching behavior in the pigeon. Journal of the Experimental Analysis of Behavior, 4, 281-284.

Drake, C. E., \& Wilson, K. G. (2008). Instructional effects on performance in a matching-to-sample study. Journal of the Experimental Analysis of Behavior, 89, 333-340. 
Dube, W. V., \& Mcllvane, W. J. (1989). Adapting a microcomputer for behavioral evaluation of mentally retarded individuals. In J. A. Mulick \& R. F. Antonack (Eds.), Transitions in mental retardation (Vol. 4, pp. 104-127). Norwood, NJ: Ablex.

Eikeseth, S., \& Smith, T. (1992). The development of functional and equivalence classes in high-functioning autistic children: the role of naming. Journal of the Experimental Analysis of Behavior, 58, 123-133.

Goyos, C., \& Almeida, J. C. (1994). Mestre 1.0 [computer software]. São Carlos: Mestre Software.

Green, G., Sigurdardottir, Z. G., \& Saunders, R. R. (1991). The role of instructions in transfer of ordinal functions through equivalence classes. Journal of the Experimental Analysis of Behavior, 55, 287-304.

Johnson, C., \& Sidman, M. (1993). Conditional discrimination and equivalence relations: control by negative stimulus. Journal of the Experimental Analysis of Behavior, 59, 333-347.

MacDonald, R. B. F., Dixon, L. S., \& LeBlanc, J. M. (1986). Stimulus class formation following observational learning. Analysis and Intervention in Developmental Disabilities, 6, 73-87.

Mcllvane, W. J., Dube, W. V., Kledaras, J. B., Iennaco, F. M., \& Stoddard, L. T. (1990). Teaching relational discrimination to individuals with mental retardation: some problems and some solutions. American Journal on Mental Retardation, 95, 283-296.

Michael, R. L., \& Bernstein, D. J. (1991). Transient effects of acquisition history on generalization in a matching to sample task. Journal of the Experimental Analysis of Behavior, 56, 155-166.

Piccolo, A. A. T. (2004). Aprendizagem observacional, formação e expansão de classes de equivalência. (Tese de doutorado, Programa de Pós-Graduação em Educação Especial, Universidade Federal de São Carlos, São Carlos, Brasil). Retirado de http://200.136.241.56/htdocs/tedeSimplificado/tde_arquivos/9/TD E-2004-12-16T07:16:56Z-353/Publico/TeseAATP.pdf

Pilgrim, C., Jackson, J., \& Galizio, M. (2000). Acquisition of arbitrary conditional discriminations by young normally developing children. Journal of the Experimental Analysis of Behavior, 73, 177-193.

Saunders, K. J., \& Spradlin, J. E. (1989). Conditional discrimination in mentally retarded adults: the effect of training the component simple discriminations. Journal of the Experimental Analysis of Behavior, 52, 1-12.

Saunders, K. J., \& Spradlin, J. E. (1993). Conditional discrimination in mentally retarded subjects: programming acquisition and learning set. Journal of the Experimental Analysis of Behavior, 60, 571-585.
Saunders, K. J., Saunders, R. R., Williams, D. C., \& Spradlin, J. E. (1993). An interaction of instructions and training design on stimulus class formation: extending the analysis of equivalence. The Psychological Record, 43, 725-744.

Sella, A. C. (2005). Ensino de discriminações condicionais por imitação: efeitos de dois arranjos de apresentação de tentativas. (Dissertação de mestrado, Programa de Pós-graduação em Educação Especial, Universidade Federal de São Carlos, São Carlos, Brasil). Retirado de http://www.bdtd.ufscar.br/htdocs/tedeSimplificado/tde_busca/pro cessaArquivo.php?codArquivo=750

Sidman, M. (1960). Tactics of Scientific Research. New York, NY: Basic Books.

Sidman, M. (1971). Reading and auditory-visual equivalence. Journal of Speech and Hearing Research, 14, 5-13.

Sidman, M. (1986). Functional analysis of emergent verbal classes. In T. Thompson \& M. D. Zeiler (Eds.), Analysis and integration of behavioral units (pp. 213-245). Hillsdale, NJ: Lawrence Erlbaum Associates.

Sidman, M. (1987). Two choices are not enough. Behavior Analysis, 22, 11-18.

Sidman, M. (1990). Equivalence relations: where do they come from? In D. E. Blackman \& H. Lejeune (Eds.), Behavior analysis in theory and practice: Contributions and controversies (pp. 93114). Hillsdale, NJ: Lawrence Erlbaum Associates.

Sidman, M. (1992). Equivalence relations: Some basic considerations. In S. C. Hayes \& L. J. Hayes (Eds.), Understanding verbal relations (pp. 15-27). Reno, NV: Context Press.

Sidman, M., \& Cresson, O. (1973). Reading and crossmodal transfer of stimulus equivalences in severe retardation. American Journal of Mental Deficiency, 77, 515-523.

Sidman, M., \& Stoddard, L. T. (1967). The effectiveness of fading in programming a simultaneous form discrimination for retarded children. Journal of the Experimental Analysis of Behavior, 10, 315.

Terrace, H. S. (1963). Errorless transfer of a discrimination across two continua. Journal of the Experimental Analysis of Behavior, 6, 223232.

Touchette, P. (1971). Transfer of stimulus control measuring the moment of transfer. Journal of the Experimental Analysis of Behavior, 15, 347-354.

Recebido em 15/04/2010

Aceito em 18/08/2011

\section{Endereço para correspondência:}

Thalita Filier Cazetto, Rua Joaquim Ulisses Sarmento, 232, Jardim Aurélia, CEP 13033-080, Campinas/SP, Brasil.E-mail: thalitacazetto@yahoo.com.br. 